\title{
Visceral adipose tissue and carotid intima- media thickness in HIV-infected patients undergoing CART: a prospective cohort study
}

Maria Teresa Beires ${ }^{1 *} \mathbb{D}$, André Silva-Pinto ${ }^{2,3}$, Ana Cristina Santos ${ }^{4,5}$, António José Madureira $^{6}$, Jorge Pereira ${ }^{7}$ Davide Carvalho ${ }^{8}$, António Sarmento ${ }^{2,3}$ and Paula Freitas ${ }^{8}$

\begin{abstract}
Background: Combined antiretroviral therapy (CART) in HIV-infected patients has been associated with lipodystrophy, metabolic abnormalities, and an increased risk of cardiovascular disease. Ultrasound measures of carotid artery intima-media thickness (CIMT) have been used as a valid measure of subclinical atherosclerosis and as a tool to predict the risk of cardiovascular events. Our aim was to evaluate the progression of cIMT in HIV-infected patients subjected to CART, with and without lipodystrophy, over a one-year period.

Methods: We performed a one-year prospective cohort study to compare changes in CIMT, metabolic and inflammation markers in HIV-infected patients undergoing CART. Body composition was assessed by dual-energy Xray absorptiometry (DXA) and abdominal computed tomography (CT). Levels of blood pressure, lipids and inflammatory markers were evaluated, as well as ultrasound measures of cIMT. Lipodystrophy defined by Fat Mass Ratio (L-FMR) is measured as the ratio of the percentage of trunk fat mass to the percentage of lower limb fat mass by DXA. Categorical variables were compared, using the chi-square or Fisher's exact test. Wilcoxon ranks tests and the McNemar chi-square tests were used to compare results of selected variables, from the first to the second year of evaluation. Means of cIMT, adjusted for age, glucose, triglycerides levels, systolic blood pressure (SBP), and waist to hip ratio were calculated, using generalised linear models for repeated measures.
\end{abstract}

Results: L-FMR was present in $44.3 \%$ of patients, and the mean of CIMT increased significantly in this group [0.82 (0.26) vs $0.92(0.33) ; p=0.037]$, as well as in patients without lipodystrophy [0.73 $(0.20)$ vs $0.84(0.30) ; p=0.012]$. In the overall sample, the progression of CIMT was statistically significant after the adjustment for age, glucose, triglycerides, and SBP, but the significance of the progression ceased after the adjustment for waist/hip ratio $[0.770(0.737-0.803)$ vs $0.874(0.815-0.933) ; p=0.514]$.

Conclusions: Carotid IMT progressed significantly in both groups of this HIV-infected cohort, however no association between the progression of cIMT and the presence of lipodystrophy defined by FMR was found. Visceral adipose tissue had an impact on the increment of cIMT, both in patients with, and without lipodystrophy defined by FMR.

Keywords: Lipodystrophy, HIV, Carotid intima media thickness, Fat mass ratio

\footnotetext{
* Correspondence: mimed11100@med.up.pt

${ }^{1}$ Faculty of Medicine, University of Porto. Alameda Prof. Hernâni Monteiro,

4200-319 Porto, Portugal

Full list of author information is available at the end of the article
} 


\section{Background}

The use of combined antiretroviral therapy (cART) in HIV-infected patients has significantly increased the life expectancy of these subjects [1]. However, as a consequence, cardiovascular disease (CVD) has emerged as being an important late concern in HIV-infected patients subjected to this treatment [2]. It has been proven that HIV-infected patients treated with cART have an increased risk of developing cardiovascular disease, and thus it became essential to understand the underlying mechanism associated with this outcome. HIVlipodystrophy is a well-established side effect of cART, especially when associated with some specific regimens [3]. Patients with lipodystrophy may present a higher risk of premature atherosclerosis, as abnormalities in body fat distribution are associated with various metabolic risk factors that lead to cardiovascular disease [4].

The relative contributions of conventional cardiovascular risk factors, metabolic side effects of antiretroviral drugs, and HIV infection itself on cardiovascular risk are difficult to identify, as these factors frequently occur simultaneously [5]. In this context, intima-media thickness of the carotid arteries (cIMT) has become a surrogate marker for atherosclerosis and it has shown to be an independent risk factor for the unfolding cardiovascular disease, on the grounds that it can be accurately and safely measured by ultrasound [6].

In a previously published study, we showed that HIVinfected patients under cART with lipodystrophy defined by FMR had a significantly higher cIMT than those without lipodystrophy, which was also associated with classical cardiovascular risk factors, such as visceral adipose tissue and age [6]. The purpose of this study was to evaluate the progression of atherosclerosis assessed by cIMT in the total of the HIV-infected patients undergoing CART, and in those with or without lipodystrophy defined by Fat Mass Ratio (L-FMR), and to evaluate the evolution of established cardiovascular risk factors during one year of follow-up.

\section{Methods}

\section{Subjects}

We evaluated 115 HIV-infected patients undergoing cART in this longitudinal study. Only noninstitutionalised caucasian adults who were subjected to cART were evaluated, and all patients were referred from the Infectious Diseases Outpatient Clinic. We excluded institutionalised patients with the objective of ruling out acute intercurrences that could have an impact on the metabolic and inflammatory parameters. Participants were evaluated at baseline, and after one year for each parameter (clinical assessment, evaluation of body composition, laboratory analysis, and carotid IMT measurements). All patients were referred to a nutrition appointment, were motivated to comply with a structured diet plan, walk or exercise regularly and were also advised to stop smoking. Overweight patients, or those suffering from obesity were motivated to lose weight. Prediabetes, diabetes, hypertension, and dyslipidemia were treated according to the standard of care for the HIV-population. This study was approved by the Ethics Committee for Health of the Hospital São João in Porto and each patient provided their written, informed consent.

\section{Clinical assessment}

For each patient, the following information was collected at both baseline and after the follow-up period (12 months), using a standard protocol that covered: age, gender, known duration of HIV infection, and duration of cART exposure, HIV risk factor, characterisation of the infection, smoking history, family history of cardiovascular diseases, and use of anti-hypertensive, anti-diabetic, or lipid lowering drugs.

We used the "Centers for Disease Control and Prevention" (CDC) HIV staging classification [7], whereby measurements of weight, height, neck, waist, hip, thigh and arm circumference were performed as previously described [8] and were carried out by the same observer, in a standard fashion [9].

Measures of blood pressure were taken with the patient in a supine position, employing a standardised technique, as previously described [10].

\section{Evaluation of body composition}

We evaluated body composition using whole-body, dual-energy X-ray absorptiometry (DXA - Lunar Expert XL, 1999). DXA measurements were carried out whilst in a supine position, and standard positioning of the arms and feet was established according to the manufacturer's instructions. Regional fat mass values were grouped and analyzed for the following anatomical regions: arms, legs, trunk, and total body. The fat mass ratio (FMR) was measured as the ratio between the percentage of trunk fat mass and the percentage of lower limb fat mass $(F M R=\%$ of the trunk fat mass $/ \%$ of the lower limb fat mass) [11]. We used a cut-off value for lipodystrophy defined by FMR for men of 1.961, and 1.329 for women [12].

The quantification of total, visceral, and subcutaneous fat was performed with a 64-slice, abdominal computed tomography (CT) scanner (Siemens Sensation 64 Cardiac), with the same technique as previously described [13, 14]. All values were expressed in $\mathrm{cm}^{2}$, rounded to the nearest centesimal. 


\section{Laboratory analysis \\ Biological and inflammatory parameters}

A venous blood sample was taken after a $12 \mathrm{~h}$ overnight fast. All the samples were analyzed at the central laboratory of our hospital. The measurements of total cholesterol (TC), low density lipoprotein (LDL) cholesterol, high-density lipoprotein (HDL) cholesterol, triglycerides, apolipoprotein A1 (Apo A1), apolipoprotein B (Apo B), lipoprotein (a) [Lp (a)], fibrinogen, high sensitivity Creactive protein (hsCRP), homocysteine, uric acid, lactates, NT-proBNP, glucose, insulin, and A1c serum levels were determined using commercial kits. Non-HDL-C was defined as TC-HDL. Microalbuminuria was determined in a 24-h urine sample. Patients without a previous diagnosis of diabetes were submitted to an oral glucose tolerance test (OGTT). This test was performed as instructed by the World Health Organisation [15].

The CD4+ cell count $\left(\times 10^{6}\right.$ cell/L) was determined by flow cytometry and plasma HIV-1 RNA loads were measured by a quantitative reverse transcriptase polymerase chain reaction (Roche Diagnostic Systems, Inc., Branchburg, NJ, USA), which had a lower limit of detection of 50 copies $/ \mathrm{mL}$.

Insulin resistance was defined by the homeostasis model assessment of insulin resistance (HOMA), using the following formula:

HOMA - IR index $=($ insulin $0 \mathrm{x}$ glucose 0$) / 22.5$ [16]

We defined Metabolic Syndrome (MS) according to the modified NCEP-ATP III criteria, requiring the presence of three of the following risk factors: (1) elevated waist circumference $(>102 \mathrm{~cm})$; (2) elevated triglycerides ( $\geq 150 \mathrm{ml} / \mathrm{dL}$ or specific treatment); (3) reduced HDL-C levels (<40 mg/dL); (4) elevated blood pressure ( $>130 /$ $85 \mathrm{mmHg}$ or treatment with anti-hypertensive drugs), and; (5) elevated fasting glucose $(\geq 100 \mathrm{mg} / \mathrm{dL}$ or use of glucose-lowering drugs). [17]

\section{Carotid IMT measurements}

We performed high-resolution B-mode and Doppler ultrasounds measures of the carotid arteries using a Philips iU22 machine (Philips Medical, The Netherlands), equipped with a 17-5 $\mathrm{MHz}$ highfrequency linear-array transducer. Patients were examined in the supine position, with the head in a neutral position, or slightly turned away from the side that was being scanned. The left and right common carotid arteries (CCA) were examined in multiple directions. The carotid bifurcation was included in the image plane to serve as a landmark and provide accurate serial measurements. The IMT was measured along a segment of the artery free of atherosclerotic plaque with clearly defined lumen-intima and media-adventitia interfaces if possible $3 \mathrm{~cm}$ below the carotid bulb. The far wall was preferred for the measurement and only when the image quality of the far wall was not satisfactory was the near wall used. The measurements were done by manual tracking and were repeated three times. The mean IMT of these values was calculated. All studies were performed by the same radiologist, who had 12 years of experience in vascular ultrasound, and were carried out using the same machine.

The presence of subclinical carotid atherosclerosis was defined as IMT $>0.80 \mathrm{~mm}$, the presence of plaque, or both [18-20].

\section{Statistical analysis}

Data were described as the mean and standard deviation (SD) for quantitative variables and were compared using Student-t or Mann-Whitney tests, as appropriate. Categorical variables were described as counts and proportions, and compared using the chi-square or Fisher's exact test. To study the association between cIMT and clinical and metabolic characteristics, Spearman correlation coefficients were estimated. In addition, Wilcoxon ranks tests and the McNemar chi-square tests were used to compare results of selected variables from the baseline to the first follow-up evaluation. Means of cIMT were calculated, using generalised linear models for repeated measures, adjusted for age, glucose, triglycerides levels, systolic blood pressure, and waist to hip ratio.

Statistical analysis was performed using SPSS version 24.0 software (SPSS Inc., Chicago, Illinois, USA). All probabilities were two-tailed, and $p$ values of $<0.05$ were regarded as significant.

\section{Results \\ Baseline \\ Patient characteristics, body composition by DXA and metabolic parameters.}

In 115 (91 male and 24 female) HIV-infected patients undergoing cART, 51 (44.3\%) presented lipodystrophy defined by FMR. The characteristics of the study sample according to the presence of lipodystrophy defined by FMR are illustrated in Table 1. Patients with lipodystrophy had been infected with HIV for a longer period of time $(p=0.036)$ and were subjected to a greater length of cART $(p=0.001)$. No differences in weight, height, BMI, waist, neck, and thigh and arm circumferences among patients with or without lipodystrophy were found.

Patients with lipodystrophy had a lower value of hip circumference and a higher waist/hip circumference ratio, compared to the patients without lipodystrophy. ( $p=0.006$ and 0.003 , respectively).

No differences were observed between patients with, and without lipodystrophy regarding viral suppression rate, HIV risk factor, CDC classification and ART regimen, smoking history, family history of cardiovascular 
Table 1 Basal sample characteristics according to the presence of lipodystrophy defined by FMR

\begin{tabular}{|c|c|c|c|}
\hline & Without Lipodystrophy defined by FMR & With Lipodystrophy defined by FMR & $P$ \\
\hline n (\%) & 64 & 51 & - \\
\hline \multicolumn{4}{|l|}{ Gender [n(\%)] } \\
\hline Male & $49(76.6 \%)$ & $42(82.4 \%)$ & \multirow[t]{2}{*}{0.448} \\
\hline Female & $15(23.4 \%)$ & $9(17.6 \%)$ & \\
\hline Age [years, mean (sd)] & $45(12.2)$ & $49(9.9)$ & 0.052 \\
\hline Duration of HIV infection [years, mean (sd)] & $7.2(3.8)$ & $8.5(3.7)$ & 0.036 \\
\hline CART [years, mean (sd)] & $5.9(3.6)$ & $7.9(3.5)$ & 0.001 \\
\hline Weight $[\mathrm{Kg}$, mean (sd)] & $69.5(13.5)$ & $67.8(9.6)$ & 0.410 \\
\hline Height [m, mean (sd)] & $1.66(0.09)$ & $1.64(0.08)$ & 0.188 \\
\hline BMI $\left[\left(\mathrm{Kg} / \mathrm{m}^{2}\right)\right.$, mean $\left.(\mathrm{sd})\right]$ & $25.2(4.7)$ & $25.2(3.8)$ & 0.976 \\
\hline Waist circumference [cm, mean (sd)] & $91.3(12.7)$ & $91.6(9.9)$ & 0.877 \\
\hline Hip circumference [cm, mean (sd)] & $95.7(8.5)$ & $91.9(6.4)$ & 0.006 \\
\hline Thigh circumference [cm, mean (sd)] & $48,7(5.1)$ & $47.1(5.2)$ & 0.113 \\
\hline Arm circumference [cm, mean (sd)] & $26.9(3.0)$ & $27.0(2.7)$ & 0.939 \\
\hline Neck circumference [cm, mean (sd)] & $37.8(3.6)$ & $37.6(3.3)$ & 0.829 \\
\hline Waist/ hip circumference ratio [mean (sd)] & $0.95(0.09)$ & $0.99(0.07)$ & 0.003 \\
\hline CD4 cell count [cells/mm3, mean (sd)] & $522.9(288.7)$ & $584.6(331.9)$ & 0.289 \\
\hline HIV RNA $(<50)[n(\%)]$ & $52(81.3 \%)$ & $47(92.92 \%)$ & 0.093 \\
\hline \multicolumn{4}{|l|}{ HIV risk factor [n (\%)] } \\
\hline Injecting drug user & $19(29.7 \%)$ & $11(21.6 \%)$ & \multirow[t]{4}{*}{0.073} \\
\hline Homosexual contact & 39 (60.9\%) & $27(52.9 \%)$ & \\
\hline Heterosexual contact & $4(6.3 \%)$ & $11(21.6 \%)$ & \\
\hline Others & $1(1.6 \%)$ & $2(3.9 \%)$ & \\
\hline \multicolumn{4}{|l|}{ CDC [n (\%)] } \\
\hline A & $32(50.0 \%)$ & $31(60.8 \%)$ & \multirow[t]{3}{*}{0.248} \\
\hline B & - & - & \\
\hline C & $32(50.0)$ & $20(39.2)$ & \\
\hline \multicolumn{4}{|l|}{ ART [n (\%)] } \\
\hline $\mathrm{Pl}$ & $32(50.0 \%)$ & $30(58.8 \%)$ & 0.346 \\
\hline NNRTI & $31(48.4 \%)$ & $23(45.1 \%)$ & 0.721 \\
\hline NRTI & $60(93.8 \%)$ & $50(98.0 \%)$ & 0.380 \\
\hline \multicolumn{4}{|l|}{ Smoking history [n (\%)] } \\
\hline Never & 19 (30.2\%) & $20(39.2)$ & \multirow[t]{3}{*}{0.556} \\
\hline Current & $33(52.4 \%)$ & $22(43.1 \%)$ & \\
\hline Former & $11(17.5 \%)$ & $9(17.6 \%)$ & \\
\hline Familial history of CVD [n (\%)] & $23(35.9 \%)$ & $21(41.2 \%)$ & 0.566 \\
\hline \multicolumn{4}{|l|}{ Taking medications [n (\%)] } \\
\hline Statins & $14(22.2 \%)$ & $15(29.4 \%)$ & 0.381 \\
\hline Fibrates & $29(46.0 \%)$ & $27(52.9 \%)$ & 0.463 \\
\hline Oral anti-diabetics & $9(14.3 \%)$ & $12(23.5 \%)$ & 0.306 \\
\hline Insulin & $4(6.3 \%)$ & $4(7.8 \%)$ & 0.999 \\
\hline Anti-hypertensive drugs & $13(20.3 \%)$ & $12(24.0 \%)$ & 0.637 \\
\hline \multicolumn{4}{|l|}{ Fat mass [\%, mean (sd)] DXA } \\
\hline Total & $22.6(13.1)$ & $17.8(8.0)$ & 0.017 \\
\hline Trunk & $24.1(13.5)$ & 22.7 (8.6) & 0.508 \\
\hline
\end{tabular}


Table 1 Basal sample characteristics according to the presence of lipodystrophy defined by FMR (Continued)

\begin{tabular}{|c|c|c|c|}
\hline & Without Lipodystrophy defined by FMR & With Lipodystrophy defined by FMR & $P$ \\
\hline Leg & $22.3(15.4)$ & $9.7(6.7)$ & $<0.001$ \\
\hline Arm & $23.4(16.3)$ & $18.7(13.3)$ & 0.095 \\
\hline \multicolumn{4}{|l|}{ Fat mass $[\mathrm{Kg}$, mean $(\mathrm{sd})]$ DXA } \\
\hline Total & $16.4(11.0)$ & $12.1(6.6)$ & 0.012 \\
\hline Trunk & $9.1(6.2)$ & $8.4(4.3)$ & 0.470 \\
\hline Leg & $4.7(3.4)$ & $1.8(1.3)$ & $<0.001$ \\
\hline Arm & $1.9(1.7)$ & $13.8(1.2)$ & 0.063 \\
\hline \multicolumn{4}{|l|}{ Body Fat Mass by Quantitative $C T$} \\
\hline Total fat $\left[\mathrm{cm}^{2}\right.$, mean $\left.(\mathrm{sd})\right]$ & $272.6(188.1)$ & $259.6(112.9)$ & 0.651 \\
\hline VAT $\left[\mathrm{cm}^{2}\right.$, mean $\left.(\mathrm{sd})\right]$ & $116.8(95.4)$ & $154.8(59.7)$ & 0.011 \\
\hline SAT $\left[\mathrm{cm}^{2}\right.$, mean $\left.(\mathrm{sd})\right]$ & $155.8(123.2)$ & $104.8(85.2)$ & 0.011 \\
\hline VAT/SAT ratio $\left[\mathrm{cm}^{2}\right.$, mean $\left.(\mathrm{sd})\right]$ & $1.3(1.8)$ & $2.4(1.7)$ & 0.002 \\
\hline Systolic blood pressure [mmHg, mean (sd)] & $122.0(19.4)$ & $124.0(16.0)$ & 0.558 \\
\hline Diastolic blood pressure $[\mathrm{mmHg}$, mean $(\mathrm{sd})]$ & $78.5(12.1)$ & $79.2(11.1)$ & 0.750 \\
\hline Leukocytes $\left[10^{9} / \mathrm{L}\right.$, mean $\left.(\mathrm{sd})\right]$ & $5.7(1.7)$ & $6.5(1.9)$ & 0.034 \\
\hline Glucose 0 min $[\mathrm{mg} / \mathrm{dL}$, mean $(\mathrm{sd})]$ & $106.4(47.1)$ & $119.1(52.1)$ & 0.175 \\
\hline Glucose 2 h [mg/dL, mean (sd)] & $122.3(46.3)$ & $138.5(47.7)$ & 0.126 \\
\hline Insulin 0 min $[\mu \mathrm{U} / \mathrm{mL}$, mean $(\mathrm{sd})]$ & $10.5(12.8)$ & $10.9(6.5)$ & 0.863 \\
\hline Insulin $2 \mathrm{~h}[\mu \mathrm{U} / \mathrm{mL}$, mean (sd)] & $53.1(49.2)$ & $105.9(186.8)$ & 0.072 \\
\hline HOMA [mean (sd)] & $2.9(3.6)$ & $3.2(2.6)$ & 0.601 \\
\hline A1c $[\%$ mean $(s d)]$ & $5.6(1.2)$ & $6.0(1.1)$ & 0.123 \\
\hline Total cholesterol [mg/dL, mean (sd)] & $213.6(56.0)$ & $226.9(57.6)$ & 0.216 \\
\hline LDL-cholesterol [mg/dL, mean (sd)] & $113.5(49.2)$ & $123.7(49.2)$ & 0.270 \\
\hline HDL-cholesterol [mg/dL, mean (sd)] & $43.9(12.1)$ & $43.6(11.3)$ & 0.911 \\
\hline Non-HDL cholesterol [mg/dL, mean (sd)] & $169.8(52.0)$ & $183.2(52.2)$ & 0.170 \\
\hline Triglycerides[mg/dL, mean (sd)] & $284.4(191.7)$ & $298.9(186.9)$ & 0.683 \\
\hline Apo A1 [mg/dL, mean (sd)] & $110.4(24.0)$ & $111.6(16.6)$ & 0.763 \\
\hline Apo B [mg/dL, mean (sd)] & $104.7(24.4)$ & $104.8(26.7)$ & 0.975 \\
\hline Ratio apo B/apo A1 [mean (sd)] & $0.98(0.3)$ & $0.95(0.3)$ & 0.604 \\
\hline Lp (a) [mg/dL, mean (sd)] & $28.9(31.0)$ & $27.5(33.5)$ & 0.821 \\
\hline Homocysteine $[\mu \mathrm{mol} / \mathrm{L}$, mean $(\mathrm{sd})]$ & $9.4(4.1)$ & $9.7(3.5)$ & 0.701 \\
\hline CRP [mg/L, mean (sd)] & $6.6(16.6)$ & $4.3(4.7)$ & 0.496 \\
\hline hsCRP [mg/dL, mean (sd)] & $0.83(1.5)$ & $0.34(0.31)$ & 0.496 \\
\hline Lactates $[\mathrm{mmol} / \mathrm{L}$, mean $(\mathrm{sd})]$ & $1.1(0.5)$ & $1.3(0.6)$ & 0.056 \\
\hline NT-ProBNP [pg/mL, mean (sd)] & $41.2(74.8)$ & $31.6(31.9)$ & 0.450 \\
\hline Fibrinogen [mg/dL, mean (sd)] & $350.8(103.8)$ & $342.9(105.9)$ & 0.696 \\
\hline Microalbumin [mg/L, mean (sd)] & 76.7 (196.6) & $40.6(91.3)$ & 0.441 \\
\hline Uric acid [mg/L, mean (sd)] & $46.0(14.6)$ & $49.8(12.8)$ & 0.140 \\
\hline Carotid IMT [mm, mean (sd)] & $0.73(0.196)$ & $0.82(0.257)$ & 0.050 \\
\hline Metabolic Syndrome (\%) & $31(49.2 \%)$ & $27(54.0 \%)$ & 0.705 \\
\hline
\end{tabular}

( $L$ Lipodystrophy FMR defined, CDC Centers for Disease Control and Prevention criteria for staging of HIV infection, CART Combined antiretroviral therapy, BMI Body mass index, FMR Fat mass ratio, CVD Cardiovascular disease, DXA Dual-energy X-ray absorptiometry, CT Computed tomography, PI Protease inhibitor, NNRTI Non-nucleoside reverse transcriptase inhibitor, NRTI Nucleoside reverse transcriptase inhibitor, VAT Visceral adipose tissue, SAT Subcutaneous adipose tissue, HOMA The homeostasis model assessment of insulin resistance, HDL High density cholesterol, LDL Low density cholesterol, Apo A1 Apolipoprotein A1, Apo B Apolipoprotein B, Lp(a) Lipoprotein (a), CPR C protein reactive, hsCRP High sensitivity C protein reactive, NT-ProBNP N-terminal prohormone of brain natriuretic peptide, IMT Intima-media thickness) 
disease and medication history (statins, fibrates, oral anti-diabetics, insulin and anti-hypertensive drugs), systolic blood pressure, and CD4+ cell count.

Regarding the assessment of body composition by DXA, patients with lipodystrophy presented lower values of total and leg fat mass $(p=0.017$ and $<0.001$, respectively), both in \% and $\mathrm{Kg}$ (Table 1 ). No differences were observed in trunk and arm fat mass (in \% and $\mathrm{Kg}$ ) between the two groups.

Patients with lipodystrophy had a higher visceral adipose tissue (VAT) value and VAT/SAT ratio $(p=0.011$ and 0.002 , respectively), lower subcutaneous adipose tissue $(\mathrm{SAT})(p=0.011)$, and no significant difference in total fat at abdominal level.

No significant differences were found in the levels of glucose, insulin, lipid profile (total cholesterol, LDL, HDL, non-HDL cholesterol and triglycerides), uric acid, lactates, HOMA, HbA1c, Apo A1, Apo B, ratio Apo B/ Apo A1, Lp(a), homocysteine, CRP, hsCRP, NT-proBNP, fibrinogen, and microalbumin urinary excretion between patients with or without lipodystrophy.

Furthermore, no significant differences regarding the prevalence of metabolic syndrome were observed between the two groups (Table 1).

\section{Carotid IMT measurements}

Carotid IMT was higher in patients with lipodystrophy, compared with those without lipodystrophy [mean (SD) $0.82(0.26)$ vs. $0.73(0.20) ; p=0.050]$ (Table 1$)$.

The cIMT was positively correlated with lipodystrophy evaluated by FMR, age, neck circumference, waist/hip ratio, systolic blood pressure, glucose at 0 and $120 \mathrm{~min}$ on OGTT, HbA1c, non-HDL cholesterol, visceral obesity defined by total body fat mass by quantitative CT, VAT and VAT/SAT ratio, trunk fat mass evaluated by DXA, uric acid, CRP, hsCRP, and homocysteine, as is shown in Table 2. No significant correlations were found between cIMT and duration of HIV infection, length of cART, thigh circumference, total, leg and arm fat mass evaluated by DXA, CD4 cell count, leukocyte, insulin at 0 and 120 min on OGTT, HOMA, triglycerides, and lactates.

The progression of the study sample's characteristics throughout the period of follow-up (12 months) is illustrated in Table 3.

\section{One year follow-up \\ Total population}

With regards to the progression of body composition and metabolic parameters during one year in the total of the sample, the values of thigh and arm circumference are lower than those obtained at baseline $(p<0.001$ and 0.049 , respectively). The systolic and diastolic blood pressure also decreased significantly in the follow-up period $(p=0.005$ and $<0.001)$, as well as total
Table 2 Associations between cIMT and body composition, as well as metabolic parameters

\begin{tabular}{|c|c|c|}
\hline Correlations between cIMT and: & $\mathrm{R}$ & $P$ \\
\hline Age & 0.709 & $<0.001$ \\
\hline Waist/Hip Ratio & 0.387 & $<0.001$ \\
\hline Neck circumference & 0.202 & 0.007 \\
\hline Systolic Blood Pressure & 0.419 & $<0.001$ \\
\hline Glucose 0 min & 0.284 & $<0.001$ \\
\hline Glucose 2 h OGTT & 0.220 & 0.006 \\
\hline A1c & 0.285 & $<0.001$ \\
\hline Non-HDL cholesterol & 0.254 & $<0.001$ \\
\hline CRP & 0.196 & 0.005 \\
\hline hsCRP & 0.334 & 0.003 \\
\hline Homocysteine & 0.150 & 0.032 \\
\hline Uric Acid & 0.146 & 0.034 \\
\hline Trunk Fat Mass by DXA (g) & 0.168 & 0.0018 \\
\hline Total fat on Abdominal CT scan & 0.218 & 0.002 \\
\hline VAT on abdominal CT scan & 0.418 & $<0.001$ \\
\hline VAT/SAT on abdominal CT scan & 0.290 & $<0.001$ \\
\hline FMR & 0.250 & $<0.001$ \\
\hline Duration of HIV infection & -0.019 & 0.779 \\
\hline Leg Fat Mass by DXA (g) & -0.037 & 0.604 \\
\hline Thigh Circumference & -0.095 & 0.169 \\
\hline CD4 cell count & -0.027 & 0.701 \\
\hline Leukocytes & -0.049 & 0.478 \\
\hline SAT on abdominal CT scan & -0.009 & 0.901 \\
\hline Insulin 2 h OGTT & -0.089 & 0.271 \\
\hline HOMA & 0.094 & 0.185 \\
\hline Insulin 0 min & 0.026 & 0.711 \\
\hline Lactates & 0.081 & 0.253 \\
\hline Triglycerides & 0.109 & 0.113 \\
\hline Total Fat Mass by DXA (g) & 0.084 & 0.236 \\
\hline Arm Fat Mass by DXA (g) & 0.029 & 0.686 \\
\hline CART & 0.024 & 0.727 \\
\hline
\end{tabular}

(FMR Fat mass ratio, OGTT Oral glucose tolerance test, CT Computed tomography, VAT Visceral adipose tissue, SAT Subcutaneous adipose tissue, $H D L$ High density cholesterol, CPR C protein reactive; hSCRP High sensitivity C protein reactive, HOMA The homeostasis model assessment of insulin resistance, DXA dual-energy X-ray absorptiometry, CART Combined antiretroviral therapy, IMT Intima-media thickness)

cholesterol, non-HDL cholesterol, and triglycerides levels $(p=0.012$ and $<0.001$, for both non-HDL cholesterol and triglycerides). The values of HDL, LDL and Apo A1 increased since the baseline $(p=0.012,0.001$ and $<0.001$, respectively), and the values of Apo B and the ratio apo $\mathrm{B} /$ apo $\mathrm{A} 1$ decreased $(p<0.001$ for both variables).

Lastly, values of homocysteine and lactates were higher $(p=0.005$ and 0.002$)$, when compared with the 
Table 3 Progression of sample characteristics during follow-up

\begin{tabular}{|c|c|c|c|c|c|c|c|c|c|}
\hline & \multicolumn{3}{|c|}{ Total Population } & \multicolumn{3}{|c|}{ With Lipodystrophy } & \multicolumn{3}{|c|}{ Without Lipodystrophy } \\
\hline & Baseline & 1 year after & $p$ & Baseline & 1 year after & $P$ & Baseline & 1 year after & $p$ \\
\hline $\begin{array}{l}\text { Weight } \\
{[\text { Kg, mean (sd) }]}\end{array}$ & $68.7(11.9)$ & $67.8(11.8)$ & 0.474 & $67.8(9.6)$ & $65.7(12.0)$ & 0.360 & $69.5(13.5)$ & $69.5(13.5)$ & 0.977 \\
\hline $\begin{array}{l}\text { Height } \\
{[\mathrm{m} \text {, mean (sd) }]}\end{array}$ & $1.65(0.1)$ & $1.65(0.1)$ & - & $1.64(0.08)$ & $1.64(0.08)$ & - & $1.66(0.09)$ & $1.66(0.09)$ & - \\
\hline $\begin{array}{l}\mathrm{BMI} \\
{\left[\left(\mathrm{Kg} / \mathrm{m}^{2}\right) \text {, mean }(\mathrm{sd})\right]}\end{array}$ & $25.2(4.3)$ & $25.0(4.9)$ & 0.547 & $25.2(3.8)$ & $24.5(4.7)$ & 0.359 & $25.2(4.7)$ & $25.4(5.1)$ & 0.849 \\
\hline $\begin{array}{l}\text { Waist circumference } \\
{[\mathrm{cm}, \text { mean }(\mathrm{sd})]}\end{array}$ & $91.4(11.5)$ & $90.9(10.8)$ & 0.654 & $91.6(9.9)$ & $89.7(11.2)$ & 0.402 & $91.4(12.8)$ & $92.0(10.4)$ & 0.804 \\
\hline $\begin{array}{l}\text { Hip circumference } \\
{[\mathrm{cm} \text {, mean }(\mathrm{sd})]}\end{array}$ & $94.0(7.8)$ & $93.2(6.8)$ & 0.496 & $91.9(6.4)$ & $92.6(7.0)$ & 0.561 & $95.7(8.6)$ & $93.6(6.7)$ & 0.120 \\
\hline $\begin{array}{l}\text { Thigh circumference } \\
{[\mathrm{cm} \text {, mean }(\mathrm{sd})]}\end{array}$ & $48.0(5.2)$ & $45.3(4.2)$ & $<0.001$ & $47.2(5.2)$ & $45.0(4.14)$ & 0.023 & $48,7(5.1)$ & $45.5(4.4)$ & $<0.001$ \\
\hline $\begin{array}{l}\text { Arm circumference } \\
{[\mathrm{cm} \text {, mean }(\mathrm{sd})]}\end{array}$ & $27.0(2.9)$ & $26.1(2.8)$ & 0.049 & $27.0(2.7)$ & $25.9(2.9)$ & 0.082 & $27.0(3.1)$ & $26.5(2.8)$ & 0.353 \\
\hline $\begin{array}{l}\text { Neck circumference } \\
{[\mathrm{cm}, \text { mean }(\mathrm{sd})]}\end{array}$ & $37.7(3.5)$ & $36.9(3.5)$ & 0.143 & $37.6(3.3)$ & $36.8(3.9)$ & 0.327 & $37.8(3.6)$ & $37.1(3.2)$ & 0.322 \\
\hline $\begin{array}{l}\text { Waist/ hip circumference ratio } \\
\text { [mean (sd)] }\end{array}$ & $0.97(0.08)$ & $0.97(0.08)$ & 0.786 & $1.00(0.07)$ & $0.97(0.09)$ & 0.086 & $0.95(0.09)$ & $0.98(0.08)$ & 0.066 \\
\hline \multicolumn{10}{|l|}{ Fat mass $[\%$, mean $(\mathrm{sd})]$ DXA } \\
\hline Total & $20.5(11.4)$ & $21.6(10.4)$ & 0.554 & $17.8(8.0)$ & $19.9(10.5)$ & 0.264 & $22.6(13.1)$ & $22.9(10.1)$ & $\begin{array}{l}0.941 \\
0.418\end{array}$ \\
\hline Trunk & $23.5(11.6)$ & $24.8(10.8)$ & 0.561 & $22.7(8.6)$ & $22.7(11.2)$ & 0.980 & $24.1(13.5)$ & $26.5(10.2)$ & \\
\hline Leg & $16.7(13.8)$ & $16.2(11.0)$ & 0.898 & $9.7(6.7)$ & $15.0(10.4)$ & 0.007 & $22.3(15.4)$ & $17.2(11.5)$ & 0.088 \\
\hline Arm & $21.3(15.2)$ & $23.8(15.0)$ & 0.267 & 18.7(13.3) & $22.4(15.8)$ & 0.232 & $23.4(16.3)$ & $24.9(14.4)$ & 0.631 \\
\hline
\end{tabular}

Fat mass

$[\mathrm{Kg}$, mean (sd)] DXA

$\begin{array}{llllllllll}\text { Total } & 14.5(9.5) & 14.7(8.3) & 0.867 & 12.1(6.7) & 13.1(7.7) & 0.406 & 16.4(11.0) & 16.0(8.6) & 0.736 \\ \text { Trunk } & 8.7(5.4) & 9.0(5.0) & 0.897 & 8.4(4.3) & 8.0(4.9) & 0.766 & 9.1(6.2) & 9.8(5.1) & 0.641 \\ \text { Leg } & 3.5(3.0) & 3.3(2.5) & 0.810 & 1.8(1.3) & 2.8(2.0) & 0.008 & 4.7(3.4) & 3.6(2.8) & 0.047 \\ \text { Arm } & 1.7(1.5) & 1.8(1.3) & 0.293 & 1.4(1.2) & 1.7(1.3) & 0.238 & 1.9(1.7) & 1.9(1.3) & 0.744\end{array}$

Body Fat Mass by Quantitative CT

Total fat

$\left[\mathrm{cm}^{2}\right.$, mean $\left.(\mathrm{sd})\right]$

VAT

$\left[\mathrm{cm}^{2}\right.$, mean (sd)]

SAT

$\left[\mathrm{cm}^{2}\right.$, mean (sd)]

VAT/SAT ratio

$\left[\mathrm{cm}^{2}\right.$, mean $\left.(\mathrm{sd})\right]$

Systolic blood pressure

$[\mathrm{mmHg}$, mean (sd)]

Diastolic blood pressure

[mmHg, mean (sd)]

Glucose 0 min

$[\mathrm{mg} / \mathrm{dL}$, mean (sd)]

Glucose $2 \mathrm{~h}$

$[\mathrm{mg} / \mathrm{dL}$, mean $(\mathrm{sd})]$

Insulin 0 min

$[\mu \mathrm{U} / \mathrm{mL}$, mean $(\mathrm{sd})]$

Insulin $2 \mathrm{~h}$

$[\mu \mathrm{U} / \mathrm{mL}$, mean $(\mathrm{sd})]$

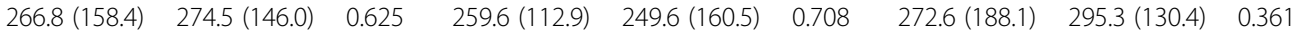

$\begin{array}{lllllllll}133.8(83.2) & 136.0(85.1) & 0.670 & 154.8(59.7) & 126.2(97.2) & 0.049 & 116.8(95.4) & 144.2(73.3) & 0.024\end{array}$

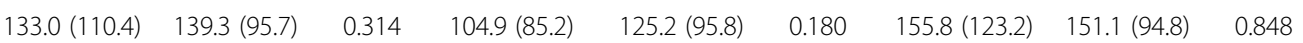

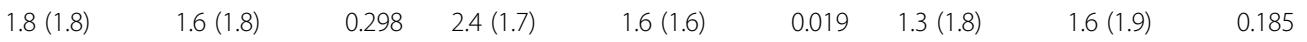

$122.9(18.0) \quad 117.2(18.1) \quad 0.005 \quad 124.0(16.1) \quad 119.3(20.9) \quad 0.072 \quad 122.0(19.4) \quad 115.6(15.5) \quad 0.027$

$\begin{array}{llllllll}78.8(11.6) & 71.8(8.9) & <0.001 & 79.2(11.1) & 72.2(8.6) & 0.001 & 78.5(12.1) & 71.6(9.2)\end{array} \quad<0.001$

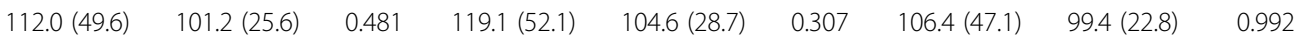

$129.5(47.4) \quad 137.3(47.6) \quad 0.445 \quad 138.5(47.7) \quad 137.7(47.5) \quad 0.819 \quad 122.3(46.3) \quad 136.9(48.2) \quad 0.451$

$\begin{array}{lllllllll}10.7(10.4) & 13.3(14.8) & 0.166 & 10.9(6.5) & 12.1(9.9) & 0.419 & 10.5(12.8) & 14.1(17.7) & 0.227\end{array}$

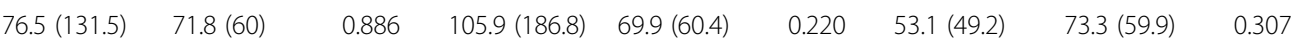


Table 3 Progression of sample characteristics during follow-up (Continued)

\begin{tabular}{|c|c|c|c|c|c|c|c|c|c|}
\hline & \multicolumn{3}{|c|}{ Total Population } & \multicolumn{3}{|c|}{ With Lipodystrophy } & \multicolumn{3}{|c|}{ Without Lipodystrophy } \\
\hline & Baseline & 1 year after & $p$ & Baseline & 1 year after & P & Baseline & 1 year after & $p$ \\
\hline $\begin{array}{l}\text { HOMA } \\
{[\text { mean (sd)] }}\end{array}$ & $3.1(3.2)$ & $3.5(4.8)$ & 0.552 & $3.2(2.5)$ & $3.2(2.9)$ & 0.857 & $2.9(3.6)$ & $3.8(5.8)$ & 0.369 \\
\hline $\begin{array}{l}\text { A1c } \\
{[\% \text { mean }(s d)]}\end{array}$ & $5.8(1.2)$ & $5.5(0.8)$ & 0.095 & $5.9(1.1)$ & $5.5(0.9)$ & 0.096 & $5.6(1.2)$ & $5.4(0.7)$ & 0.556 \\
\hline $\begin{array}{l}\text { Total cholesterol } \\
{[\mathrm{mg} / \mathrm{dL} \text {, mean (sd)] }}\end{array}$ & $219.5(56.8)$ & $204.7(41.4)$ & 0.012 & $226(57.6)$ & $203.8(41.9)$ & 0.007 & $213.6(56.0)$ & $205.4(41.2)$ & 0.396 \\
\hline $\begin{array}{l}\text { LDL-cholesterol } \\
{[\mathrm{mg} / \mathrm{dL} \text {, mean (sd)] }}\end{array}$ & $118.0(49.2)$ & $135.7(31.3)$ & 0.001 & $123.7(49.2)$ & $133.9(28.4)$ & 0.153 & $113.5(49.2)$ & $137.2(33.6)$ & 0.001 \\
\hline $\begin{array}{l}\text { HDL-cholesterol } \\
{[\mathrm{mg} / \mathrm{dL} \text {, mean }(\mathrm{sd})]}\end{array}$ & $43.8(11.7)$ & $48.6(13.3)$ & 0.012 & $43.6(11.3)$ & $50.6(14.6)$ & 0.018 & $43.9(12.1)$ & $46.9(12.0)$ & 0.280 \\
\hline $\begin{array}{l}\text { Non-HDL cholesterol } \\
{[\mathrm{mg} / \mathrm{dL} \text {, mean }(\mathrm{sd})]}\end{array}$ & $175.7(52.3)$ & $156.2(37.6)$ & $<0.001$ & $183.2(52.2)$ & $153.2(37.6)$ & $<0.001$ & $169.8(52.0)$ & $158.5(37.6)$ & 0.192 \\
\hline $\begin{array}{l}\text { Triglycerides } \\
{[\mathrm{mg} / \mathrm{dL} \text {, mean (sd)] }}\end{array}$ & $290.8(189.0)$ & $180.5(110.5)$ & $<0.001$ & $298.9(186.9)$ & $165.6(107.2)$ & $<0.001$ & $284.4(191.7)$ & $192.3(112.5)$ & 0.001 \\
\hline $\begin{array}{l}\text { Apo A1 } \\
{[\mathrm{mg} / \mathrm{dL} \text {, mean (sd)] }}\end{array}$ & $111(21)$ & $126.4(24.0)$ & $<0.001$ & $111.6(16.6)$ & $130.0(25.2)$ & 0.001 & $110.4(23.9$ & $123.7(22.8)$ & 0.002 \\
\hline $\begin{array}{l}\text { Apo B } \\
{[\mathrm{mg} / \mathrm{dL} \text {, mean }(\mathrm{sd})]}\end{array}$ & $104.7(25.3)$ & $101.4(21.7)$ & $<0.001$ & $104.8(26.7)$ & $100.0(21.6)$ & 0.126 & $104.7(24.4)$ & $102.4(22.0)$ & 0.706 \\
\hline $\begin{array}{l}\text { Ratio apo B/apo A1 } \\
\text { [mean (sd)] }\end{array}$ & $0.97(0.3)$ & $0.83(0.3)$ & $<0.001$ & $0.95(0.3)$ & $0.79(0.2)$ & 0.001 & $0.98(0.3)$ & $0.87(0.3)$ & 0.02 \\
\hline $\begin{array}{l}\operatorname{Lp}(\mathrm{a}) \\
{[\mathrm{mg} / \mathrm{dL} \text {, mean }(\mathrm{sd})]}\end{array}$ & $28.3(32.0)$ & $36.3(43.3)$ & 0.289 & $27.5(33.5$ & $44.4(47.8)$ & 0.102 & 28.9 (30.9) & $30.1(38.7)$ & 0.906 \\
\hline $\begin{array}{l}\text { Homocysteine } \\
{[\mu \mathrm{mol} / \mathrm{L} \text {, mean (sd)] }}\end{array}$ & 9.5 (3.8) & $11.1(4.8)$ & 0.005 & 9.7 (3.5) & $12.1(5.2)$ & 0.008 & $9.4(4.1)$ & $10.3(4.3)$ & 0.179 \\
\hline $\begin{array}{l}\text { CRP } \\
{[\mathrm{mg} / \mathrm{L} \text {, mean }(\mathrm{sd})]}\end{array}$ & $5.6(12.8)$ & $5.1(11.3)$ & 0.147 & $4.3(4.7)$ & $6.1(14.4)$ & 0.857 & $6.6(16.6)$ & $4.3(8.0)$ & 0.038 \\
\hline $\begin{array}{l}\text { hsCRP } \\
{[\mathrm{mg} / \mathrm{dL} \text {, mean (sd)] }}\end{array}$ & $0.6(1.2)$ & $0.5(1.2)$ & 0.030 & $0.34(0.31)$ & $0.65(1.6)$ & 0.225 & $0.83(1.5)$ & $0.43(0.8)$ & 0.066 \\
\hline $\begin{array}{l}\text { Lactates } \\
{[\mathrm{mmol} / \mathrm{L} \text {, mean }(\mathrm{sd})]}\end{array}$ & $1.2(0.5)$ & $1.4(0.6)$ & 0.002 & $1.3(0.6)$ & $1.4(0.6)$ & 0.307 & $1.1(0.48)$ & $1.4(0.5)$ & 0.001 \\
\hline $\begin{array}{l}\text { NT-ProBNP } \\
{[\mathrm{pg} / \mathrm{mL} \text {, mean (sd)] }}\end{array}$ & $36.8(59.0)$ & $39.0(63.0)$ & 0.241 & 31.6 (31.9) & $35.9(33.2)$ & 0.177 & $41.2(74.8)$ & $41.3(79.0)$ & 0.769 \\
\hline $\begin{array}{l}\text { Fibrinogen } \\
{[\mathrm{mg} / \mathrm{dL} \text {, mean (sd)] }}\end{array}$ & $347.3(104.4)$ & $332.6(79,0)$ & 0.611 & $342.9(105.9)$ & $333.8(80.1)$ & 0.841 & $350.8(103.8)$ & $331.6(78.8)$ & 0.370 \\
\hline $\begin{array}{l}\text { Microalbumin } \\
{[\mathrm{mg} / \mathrm{L} \text {, mean (sd)] }}\end{array}$ & $58.2(151.4)$ & $36.0(159.8)$ & 0.527 & $40.6(91.3)$ & 20.5 (35.6) & 0.551 & 76.7 (196.6) & $47.7(209.3)$ & 0.852 \\
\hline $\begin{array}{l}\text { Uric acid } \\
{[\mathrm{mg} / \mathrm{L} \text {, mean (sd)] }}\end{array}$ & $47.7(14.0)$ & 44.1 (14.6) & 0.022 & $49.8(12.8)$ & 43.7 (13.0) & 0.005 & 45.9 (14.6) & $474.3(15.8)$ & 0.470 \\
\hline $\begin{array}{l}\text { Carotid IMT } \\
{[\mathrm{mm}, \text { mean (sd)] }}\end{array}$ & $0.77(0.23)$ & $0.87(0.32)$ & 0.001 & $0.82(0.26)$ & $0.92(0.33)$ & 0.037 & $0.73(0.20)$ & $0.84(0.30)$ & 0.012 \\
\hline Metabolic Syndrome (\%) & $58(51.3)$ & $48(43.2)$ & 0.203 & $27(54.0)$ & 19 (38.8) & 0.134 & $31(49.2)$ & $29(46.8)$ & 0.851 \\
\hline
\end{tabular}

(L- Lipodystrophy FMR defined; BMI Body mass index, FMR Fat mass ratio, DXA Dual-energy X-ray absorptiometry, CT Computed tomography, VAT Visceral adipose tissue, SAT Subcutaneous adipose tissue, HOMA The homeostasis model assessment of insulin resistance, HDL High density cholesterol, LDL Low density cholesterol, Apo A1 Apolipoprotein A1, Apo B Apolipoprotein B, $L p(a)$ Lipoprotein (a), CPR C protein reactive, hsCRP High sensitivity C protein reactive, NT-ProBNP N-terminal prohormone of brain natriuretic peptide $I M T$ Intima-media thickness)

baseline, and the values of hsCRP and uric acid were lower $(p=0.030$ and 0.022$)$.

\section{Patients with Lipodystrophy}

Patients with lipodystrophy presented lower thigh circumference when compared with the baseline ( $p=$ 0.023), but higher values of leg fat mass, both in $\mathrm{Kg}$ and $\%(p=0.008$ and 0.007 , respectively). The analysis according to quantitative $\mathrm{CT}$ showed a decreased value of VAT and VAT/SAT ratio $(p=0.049$ and 0.019$)$. Diastolic blood pressure, total cholesterol, non-HDL cholesterol and triglycerides levels were lower compared with baseline values $(p=0.001,0.007$, and $<0.001$ for both non HDL-cholesterol and triglycerides levels). Furthermore, 
HDL and Apo A1 levels increased ( $p=0.018$ and 0.001), and the Apo B/Apo A1 ratio decreased $(p=0.001)$. The values of homocysteine were higher, while uric acid levels were lower than the ones obtained at the baseline $(p=0.008$ and 0.005 , respectively).

\section{Patients without Lipodystrophy}

Patients without lipodystrophy presented lower thigh circumference and leg fat mass $(\mathrm{Kg})$ compared with the baseline $(p<0.001$ and 0.047$)$. The value of VAT increased in this group during the follow-up period ( $p=$ $0.024)$. In addition to the decline of systolic and diastolic blood pressure ( $p=0.027$ and $<0.001$, respectively), CRP and hsCRP levels also decreased ( $p=0.038$ and 0.066). The values of LDL, Apo A1 and lactates increased ( $p=$ $0.001,0.002$ and 0.001), while triglycerides levels and Apo B/Apo A1 ratio decreased $(p=0.001$ and 0.02 , respectively).

\section{Carotid IMT measurements}

During one year of follow-up there was an increase in carotid IMT in the total of HIV-infected patients and in those with and without lipodystrophy, when compared with the baseline $(p=0.001,0.037$ and 0.012 , respectively), and this remained higher in patients presenting lipodystrophy when compared to those without lipodystrophy, although without significant differences between them $(p=0.178)$.

Table 2 shows the variables that were positively correlated with carotid IMT at the baseline. The one year evolution of these variables during follow-up in the total population can be observed in Table 4. During the follow-up period, the cIMT increased in the totality of the sample $[0.77(0.23)$ and 1 year after $0.87(0.32) \mathrm{mm}$; $p=0.001]$, despite the significant decrease in the values of systolic blood pressure [122.9 (18.0) and 1 year after 117.2 (18.1) $\mathrm{mmHg} ; p=0.005]$, diastolic blood pressure [78.8 (11.6) and 1 year after 71.8 (8.9) mmHg; $p<0.001]$, total cholesterol $[219.5$ (56.8) and 1 year after 204.7 (41.4) $\mathrm{mg} / \mathrm{dL} ; p=0.012]$, non-HDL cholesterol [175.7 (52.3) and 1 year after $156.2(37.6) \mathrm{mg} / \mathrm{dL} ; \mathrm{p}<0.001]$, triglycerides [290.8 (189.0) and 1 year after 180.5 (110.5) $\mathrm{mg} / \mathrm{dL} ; \mathrm{p}<0.001]$, apo B [104.7 (25.3) and 1 year after 101.4 (21.7) $\mathrm{mg} / \mathrm{dL} ; \mathrm{p}<0.001]$, hsCRP [0.6 (1.2) and 1 year after $0.5(1.2) \mathrm{mg} / \mathrm{dL} ; p=0.030$ ] and uric acid [47.7 (14.0) and 1 year after 44.1 (14.6) $\mathrm{mg} / \mathrm{L} ; p=0.022$ ]. The only variable positively correlated with cIMT that significantly increased in the total population was homocysteine.

Using generalised linear models for repeated measures, means of cIMT between the baseline and the 1 year follow-up were calculated and adjusted for age, glucose,

Table 4 Evolution of the variables positively correlated with cIMT during follow-up in the total sample

\begin{tabular}{|c|c|c|c|}
\hline & Baseline & One Year follow-up & $P$ \\
\hline Waist/Hip Ratio [mean (sd)] & $0.97(0.08)$ & $0.97(0.08)$ & 0.786 \\
\hline $\begin{array}{l}\text { Neck circumference } \\
{[\mathrm{cm}, \text { mean }(\mathrm{sd})]}\end{array}$ & $37.7(3.5)$ & $36.9(3.5)$ & 0.143 \\
\hline $\begin{array}{l}\text { Systolic Blood Pressure } \\
{[\mathrm{mmHg} \text {, mean (sd)] }}\end{array}$ & $122.9(18.0)$ & $117.2(18.1)$ & 0.005 \\
\hline Glucose 0 min $[\mathrm{mg} / \mathrm{dL}$, mean (sd)] & $112.0(49.6)$ & $101.7(25.6)$ & 0.481 \\
\hline $\begin{array}{l}\text { Glucose } 2 \text { h OGTT } \\
{[\mathrm{mg} / \mathrm{dL} \text {, mean (sd)] }}\end{array}$ & $129.5(47.4)$ & $137.3(47.6)$ & 0.445 \\
\hline A1c $[\%$ mean $(s d)]$ & $5.8(1.2)$ & $5.5(0.8)$ & 0.095 \\
\hline $\begin{array}{l}\text { Non-HDL cholesterol } \\
{[\mathrm{mg} / \mathrm{dL} \text {, mean }(\mathrm{sd})]}\end{array}$ & $175.7(52.3)$ & $156.2(37.6)$ & $<0.001$ \\
\hline CRP $[\mathrm{mg} / \mathrm{L}$, mean $(\mathrm{sd})]$ & $5.6(12.8)$ & $5.1(11.3)$ & 0.147 \\
\hline hsCRP $[\mathrm{mg} / \mathrm{dL}$, mean (sd)] & $0.6(1.2)$ & $0.5(1.2)$ & 0.030 \\
\hline Homocysteine $[\mu \mathrm{mol} / \mathrm{L}$, mean $(\mathrm{sd})]$ & $9.5(3.8)$ & $11.1(4.8)$ & 0.005 \\
\hline Uric Acid [mg/L, mean (sd)] & $47.7(14.0)$ & $44.1(14.6)$ & 0.022 \\
\hline $\begin{array}{l}\text { Trunk Fat Mass by DXA }(\mathrm{kg}) \\
{[\mathrm{Kg} \text {, mean }(\mathrm{sd})]}\end{array}$ & $8.7(5.4)$ & $9.0(5.0)$ & 0.897 \\
\hline $\begin{array}{l}\text { Total fat on Abdominal } \\
\mathrm{CT} \mathrm{scan}\left[\mathrm{cm}^{2} \text {, mean (sd)] }\right.\end{array}$ & $266.8(158.4)$ & $274.5(146.0)$ & 0.625 \\
\hline $\begin{array}{l}\text { VAT on abdominal } \\
C T \text { scan }\left[\mathrm{cm}^{2} \text {, mean (sd)] }\right.\end{array}$ & $133.8(83.2)$ & $136.0(85.1)$ & 0.670 \\
\hline $\begin{array}{l}\text { VAT/SAT on abdominal } \\
C T \text { scan }\left[\mathrm{cm}^{2} \text {, mean }(\mathrm{sd})\right]\end{array}$ & $1.8(1.8)$ & $1.6(1.8)$ & 0.298 \\
\hline
\end{tabular}

OGTT Oral glucose tolerance test, CT Computed tomography, VAT Visceral adipose tissue, SAT Subcutaneous adipose tissue, HDL High density cholesterol, CPR C protein reactive, $h S C R P$ High sensitivity $C$ protein reactive, DXA Dual-energy X-ray absorptiometry, IMT Intima-media thickness) 
triglycerides levels, systolic blood pressure and waist to hip ratio (Table 5). In the total population, significantly higher mean values of cIMT were observed after the one year follow-up, after adjustment for age [0.770 (0.738$0.802)$ basal vs $0.874(0.816-0.9321$ year after, <0.001], for age and glucose [0.770 $(0.737-0.803)$ basal vs 0.874 (0.816-0.932) 1 year after; $p<0.001$ ], for age, glucose and triglycerides $[0.770(0.737-0.803)$ basal vs 0.874 (0.815-0.933) 1 year after; $p=0.001]$ and for age, glucose, triglycerides, and systolic blood pressure $[0.770$ (0.738-0.802) basal vs 0.874 (0.815-0.932) 1 year after; $p=0.050]$. However, after further adjustment for age, glucose, triglycerides, systolic pressure, and waist/hip ratio, this difference did not remain statistically significant $[0.770(0.737-0.803)$ basal vs $0.874(0.815-0.933)$ 1 year after; $p=0.514]$.

The same analysis was performed stratifying for the presence of lipodystrophy, and higher mean adjusted values of cIMT [0.817 (0.744-0.889) vs $0.919(0.825-1.01)$
1 year after; $p=0.030]$, adjusted for age [0.817 (0.757$0.876)$ vs $0.919(0.826-1.01) ; p=0.027)]$, age and glucose [0.817 (0.756-0.877) vs $0.919(0.825-1.01) ; p=0.018]$, and age, glucose and triglycerides [0.817 (0.756-0.877) vs $0.919(0.825-1.01) ; p=0.053)$ were observed at one year of follow-up, but this difference lost its statistical significance after further adjustment for systolic blood pressure [0.817 (0.756-0.878) vs $0.91(0.825-1.01) ; p=0.401)$, and waist/hip ratio [0.817 (0.755-0.878) vs $0.919(0.823-1.01)$; $p=0.644]$. The same results were observed for patients without lipodystrophy (Table 5).

\section{Discussion}

Atherosclerosis is a progressive disease, and its contribution to cardiovascular risk increase is well-known [21]. This conventional CVD risk factor, as well as body fat redistribution, is associated with the progression of cIMT [22]. The intervention in cardiometabolic risk factors through the introduction of changes in terms of

Table 5 Crude and adjusted means of cIMT at baseline and at 1 year follow up

\begin{tabular}{|c|c|c|c|}
\hline Total Sample & cIMT baseline & cIMT 1 year follow up & $P$ \\
\hline Crude model & $0.770(0.728-0.812)$ & $0.874(0.815-0.932)$ & $<0.001$ \\
\hline Model adjusted for age & $0.770(0.738-0.802)$ & $0.874(0.816-0.932)$ & $<0.001$ \\
\hline Model adjusted for age and glucose & $0.770(0.737-0.803)$ & $0.874(0.816-0.932)$ & $<0.001$ \\
\hline $\begin{array}{l}\text { Model adjusted for age, glucose and } \\
\text { Triglycerides }\end{array}$ & $0.770(0.737-0.803)$ & $0.874(0.815-0.933)$ & 0.001 \\
\hline $\begin{array}{l}\text { Model adjusted for age, glucose, triglycerides } \\
\text { and Systolic Pressure }\end{array}$ & $0.770(0.738-0.802)$ & $0.874(0.815-0.932)$ & 0.050 \\
\hline $\begin{array}{l}\text { Model adjusted for age, glucose, triglycerides, } \\
\text { Systolic Pressure and WHR }\end{array}$ & $0.770(0.737-0.803)$ & $0.874(0.815-0.933)$ & 0.514 \\
\hline \multicolumn{4}{|l|}{ Patients with Lipodystrophy } \\
\hline Crude model & $0.817(0.744-0.889)$ & $0.919(0.825-1.01)$ & 0.030 \\
\hline Model adjusted for age & $0.817(0.757-0.876)$ & $0.919(0.826-1.01)$ & 0.027 \\
\hline Model adjusted for age and glucose & $0.817(0.756-0.877)$ & $0.919(0.825-1.01)$ & 0.018 \\
\hline $\begin{array}{l}\text { Model adjusted for age, glucose and } \\
\text { triglycerides }\end{array}$ & $0.817(0.756-0.877)$ & $0.919(0.825-1.01)$ & 0.053 \\
\hline $\begin{array}{l}\text { Model adjusted for age, glucose, triglycerides } \\
\text { and Systolic Pressure }\end{array}$ & $0.817(0.756-0.878)$ & $0.919(0.825-1.01)$ & 0.401 \\
\hline $\begin{array}{l}\text { Model adjusted for age, glucose, triglycerides, } \\
\text { Systolic Pressure and WHR }\end{array}$ & $0.817(0.755-0.878)$ & $0.919(0.823-1.01)$ & 0.644 \\
\hline \multicolumn{4}{|l|}{$\begin{array}{l}\text { Patients Without } \\
\text { Lipodystrophy }\end{array}$} \\
\hline Crude model & $0.733(0.684-0.784)$ & $0.838(0.763-0.914)$ & 0.020 \\
\hline Model adjusted for age & $0.733(0.698-0.767)$ & $0.838(0.763-0.914)$ & 0.003 \\
\hline Model adjusted for age and glucose & $0.733(0.698-0.768)$ & $0.838(0.763-0.914)$ & 0.015 \\
\hline $\begin{array}{l}\text { Model adjusted for age, glucose and } \\
\text { triglycerides }\end{array}$ & $0.733(0.698-0.768)$ & $0.838(0.762-0.915)$ & 0.017 \\
\hline $\begin{array}{l}\text { Model adjusted for age, glucose, triglycerides } \\
\text { and Systolic Pressure }\end{array}$ & $0.733(0.698-0.768)$ & $0.838(0.762-0.915)$ & 0.130 \\
\hline $\begin{array}{l}\text { Model adjusted for age, glucose, triglycerides, } \\
\text { Systolic Pressure and WHR }\end{array}$ & $0.733(0.698-0.768)$ & $0.838(0.761-0.915)$ & 0.676 \\
\hline
\end{tabular}

(L- Lipodystrophy FMR defined; FMR Fat mass ratio, SBP Systolic blood pressure, WHR Waist/hip ratio, IMT Intima-media thickness) 
diet, exercise, smoking cessation, and drug therapy (antidiabetic, antihypertensive and anti-dyslipidemic) may regress or delay the progression of atherosclerosis [23]. We evaluated the progression of cIMT at the end of the one year follow-up in a cohort of HIV-infected patients under cART, treated for the conventional risk factors. Indeed, after a year of follow-up, patients improved some lipid profile components and blood pressure levels, and showed decreased VAT values. Despite these improvements, mean values of cIMT increased during this period of time. In fact, after one year, HIV-infected patients (with and without lipodystrophy) had a significant progression of cIMT, even after adjustment for some conventional cardiovascular risk factors (age, glucose and triglycerides). However, during the follow-up, we observed an increase in LDL in the total of the population and also in those without lipodystrophy, which could impact on the progression of cIMT.

Furthermore, the presence of lipodystrophy defined by FMR was not associated with the progression of cIMT. Our data suggest that the increase of cIMT is associated with body fat abnormalities, particularly visceral body fat. It has been established that cART can cause a wide spectrum of metabolic disturbances and abnormalities in body fat distribution, and its contribution to premature and accelerated atherosclerosis in HIV infected patients is well-known [10, 24, 25]. Since our main goal was to determine whether the progression of cIMT was associated with lipodystrophy, our sample only included HIV infected patients under cART. The overall progression of cIMT in both groups (with and without lipodystrophy) may reflect the fact that all the patients were subjected to cART and all were under a low-grade chronic state of inflammation associated with the HIV disease per se.

As pointed out above, the mean values of cIMT increased significantly in both groups of the population, despite the fact that, at the baseline, lipodystrophic patients had a higher waist/hip circumference ratio, VAT and VAT/SAT ratio. At follow-up, on the other hand, this group had lower WHR values, but showed equivalent results for cardiometabolic markers, when compared with patients without lipodystrophy. During the one year of follow-up, the values for VAT, VAT/ SAT ratio, and WHR did not change significantly in the total of the population, and neither in those with and without lipodystrophy. Analyses from large observational cohorts have demonstrated that cIMT increase ranging from 0.03 to $0.2 \mathrm{~mm}$ are associated with $33 \%$ to $300 \%$ higher risk of coronary heart disease and stroke [26-28]. In our study, the progression of cIMT was over $0.1 \mathrm{~mm}$ in both groups, thus reflecting an increased cardiovascular risk in HIV infected patients under cART.
The degree to which cART therapy contributes to the increased cardiovascular risk in HIV patients is not completely clear yet. Even though there is a large body of evidence in the literature pointing to a moderate association between HIV infection and increased cIMT, in both cross-sectional and prospective studies, the results are still controversial [29-31]. A prospective study that evaluated the progression of cIMT in HIV-infected patients during 6 years found that HIV-infected patients accumulate CVD risk over time, beyond that experienced in the general population [32]. A recent large prospective cohort study that evaluated the progression of subclinical atherosclerosis in HIV-infected patients, over seven years, concluded that HIV infection is associated with increased cardiovascular risk and that this elevated risk persisted among cART-treated individuals with persistent HIV viral suppression [33]. Hanna et al. showed that HIV infection is associated with greater increases in focal plaque formation, even when the patients are subjected to antiretroviral therapy, but that HIV infection itself was not associated with increased cIMT over time, and no associations were found between persistent virologic suppression and cIMT progression, compared with uninfected individuals [33]. These findings contradict several other studies that reported an association between HIV infection and cIMT progression over time [5, 32, 34-37]. Although van Vonderen et al. reported an independent association between HIV infection and increased cIMT, cART use was only associated with increased stiffness of the femoral artery, not cIMT [5]. The same study also did not find an independent association between lipodystrophy and cIMT [5], which is consistent with our study. Volpe et al. found that changes in cIMT over time were associated not only with tradition risk factors, but also with HIV-related factors and that these associations vary with the type of surrogate marker evaluated [30]. In fact, some studies have suggested that traditional risk factors overshadow the role of HIV infection and cART use, and that they are the major influences of cIMT progression [6, 37-39]. Visceral obesity is a well-known independent risk factor for CVD in terms of the population as a whole, [40] and independent associations between carotid intima-media thickness and abdominal adiposity were already demonstrated [41]. Although the physiologic mechanisms by which obesity is linked to an early carotid atherosclerosis are not clearly described, some theories regarding the importance of metabolic factors, such as changes in plasma adiponectin levels and insulin resistance, have been proposed [6]. In our study, we found a higher prevalence of visceral adiposity among patients with lipodystrophy. We expected that the visceral adiposity present in patients with lipodystrophy would contribute to the progression of cIMT, which was not the case. We 
can speculate that the high frequency of visceral adiposity in patients without lipodystrophy defined by FMR may contribute to the absence of differences in the progression of cIMT between patients with and without lipodystrophy. In contrast with the results obtained at baseline, the mean values of cIMT remained significant after adjustment for age. In fact, the progression of cIMT remained significant after adjustment for age, glucose, triglycerides and systolic blood pressure, but statistical significance was lost when the association was further adjusted for the waist/hip ratio. This indicates that although cIMT is known to be affected by age, in our study this was not the case, as, in fact, cIMT progression was mostly affected by the presence of visceral adiposity.

Additional research, with a large sample-size, is needed to better characterize the underlying proatherogenic mechanisms of HIV infection and cART, as well as to evaluate the impact of body composition, namely lipodystrophy, on long-term cardiovascular risk in HIVinfected patients.

\section{Study limitations}

The limitations of this study are mainly related to the small size of the sample. Therefore, this study might have been underpowered for the detection of small differences in cIMT progression between the two groups. Due to the fact that the design of this study did not include an HIV-uninfected control group, it remains unclear whether the observed increased cardiovascular risk can be fully explained by the effects of antiretroviral drugs and their metabolic side effects, body composition, or whether chronic HIV infection itself may play a role. Another limitation of our study is the absence of data regarding the exposure time to each drug, since we only have information on the current class of antiretroviral, thus the impact of the duration of cART regimen could not be assessed in this study.

\section{Highlights of this study}

This study emphasizes the role of body composition, namely VAT, in addition to other traditional risk factors in the progression of cIMT. Moreover, it was performed in a unit that is highly experienced in the assessment of metabolic and body fat abnormalities in HIV-infected patients. All clinical evaluations were carried out by the same practitioner, and an objective definition of lipodystrophy was used (fat mass ratio by DXA).

\section{Conclusions}

Carotid IMT progressed significantly in HIV-infected patients under cART, both in those with and without lipodystrophy. Although patients with lipodystrophy defined by FMR had a higher cIMT when compared with those without lipodystrophy, there was no association between the progression of cIMT and the presence of lipodystrophy defined by FMR. Visceral adipose tissue had an impact on the increment of cIMT during the follow-up period (both in patients with and without lipodystrophy defined by FMR), which suggests an independent association between VAT and the increasing of subclinical carotid atherosclerosis.

\begin{abstract}
Abbreviations
CART: Combination antiretroviral therapy; CIMT: Carotid intima-media thickness; CVD: Cardiovascular disease; FMR: Fat Mass Ratio; L-FMR: Lipodystrophy defined by FMR; OGTT: Oral glucose tolerance test; SAT: Subcutaneous adipose tissue; SPB: Systolic blood pressure; VAT: Visceral adipose tissue; WHR: Waist/hip ratio
\end{abstract}

\section{Acknowledgments}

Not applicable.

\section{Funding}

Research Fellowship Dr. Manuel Almeida Ruas from the Portuguese Society of Diabetology. Research Fellowship from the Portuguese Association for Clinical Study of AIDS. Research Grant to support doctoral studies in the area of HIV/AIDS. GlaxoSmithKline Foundation of Health Sciences. ACS holds a FCT Investigator contract IF/01060/2015.

\section{Availability of data and materials}

The data that support the findings of this study are available on request from the corresponding author [MTB]. The data are not publicly available because they contain information that could compromise research participant privacy/consent.

\section{Authors' contributions}

MTB participated in the acquisition of data and drafted the manuscript; ASP participated in the acquisition of data and revised the manuscript; ACS performed the statistical analysis and critically revised the manuscript; AJM performed the CT scans and reviewed the data; JP performed DXA scans and reviewed the data; DC conceived the study and participated in its design; AS critically revised the manuscript; PF conceived the study, participated in its design, in the acquisition of data and critically revised the manuscript. All authors read and approved the final manuscript.

\section{Ethics approval and consent to participate}

All patients provided written informed consent and the study protocol was approved by the São João Hospital's Ethics Committee for Health.

Consent for publication

Not applicable.

Competing interests

The authors declare that they have no competing interests.

\section{Publisher's Note}

Springer Nature remains neutral with regard to jurisdictional claims in published maps and institutional affiliations.

\section{Author details}

${ }^{1}$ Faculty of Medicine, University of Porto. Alameda Prof. Hernâni Monteiro, 4200-319 Porto, Portugal. ${ }^{2}$ Infectious Diseases Department, Centro Hospitalar São João, Porto, Portugal. ${ }^{3}$ Renal, Urological and Infectious Diseases Department, Faculty of Medicine of University of Porto, Porto, Portugal. ${ }^{4}$ EPIUnit - Instituto de Saúde Pública, Universidade do Porto, Porto, Portugal. ${ }^{5}$ Departamento de Ciências da Saúde Pública e Forenses e Educação Médica, Faculdade de Medicina, Universidade do Porto, Porto, Portugal. ${ }^{6}$ Radiology Department, Hospital de São João and University of Porto Medical School, Porto, Portugal. ${ }^{7}$ Nuclear Medicine Department, Hospital de São João, Porto, Portugal. ${ }^{8}$ Endocrinology Department, Hospital de São João and University of Porto Medical School, Porto, Portugal. 
Received: 15 June 2017 Accepted: 5 December 2017

\section{Published online: 11 January 2018}

\section{References}

1. Hogg R, Lima V, Sterne JA, Grabar S, Battegay M, Bonarek M, D'Arminio Monforte A, Esteve A, Gill MJ, Harris R, Justice A, Hayden A, Lampe F, Mocroft A, Mugavero MJ, Staszewski S, Wasmuth JC, van Sighem A, Kitahata M, Guest J, Egger M, May M. Life expectancy of individuals on combination antiretroviral therapy in high-income countries: a collaborative analysis of 14 cohort studies. Lancet. 2008;372:293-9.

2. Palella FJ, Delaney KM, Moorman AC, Loveles MO, Fuher J, SattenGA, Aschman DJ, Holmberg SD: Declining morbidity and mortality among patients with advanced human immunodeficiency virus infection: HIV outpatient study investigators. N Engl J Med 1998;338:853-860.

3. Lichtenstein KA. Redefining lipodystrophy syndrome: risks and impact on clinical decision making. J Acquir Immune Defic Syndr. 2005;39(4):395-400.

4. Brown TT, Li X, Cole SR, Kingsley LA, Palella FJ, Riddler SA, Chimel JS, Visscher BR, Margolick JB, Dobs AS. Cumulative exposure to nucleoside analogue reverse transcriptase inhibitors is associated with insulin resistance markers in the multicenter AIDS cohort study. AIDS. 2005;19(13):1375-83.

5. van Vonderen MGA, Smulders YM, Stehouwer CD, Danner SA, Gundy CM, Vos F, Reiss P, van Agtmael MA. Carotid intima-media thickness and arterial stiffness in HIV-infected patients: the role of HIV, antiretroviral therapy and Lipodystrophy. J Acquir Immune Defic Syndr. 2009;50:153-61.

6. Freitas P, Carvalho D, Santos AC, Madureira AJ, Martinez E, Pereira J, Sarmento A, Medina JL. Carotid intima media thickness is associated with body fat abnomarlities in HIV-infected patients. BMC Infect Dis. 2014;14:348-56

7. Castro KG, Ward JW, Slutsker L, Buehler JW, Jaffe HW, Berkelman RL. National Center for infectious diseases division of HIV/AIDS: 1993 revised classification system for HIV infection and expanded surveillance case definition for AIDS among adolescents and adults. Morb Mortal Wkly Rep. 1992:41:1-19.

8. Freitas P, Carvalho D, Santos AC, Mesquita J, Correia F, Xerinda S, Marques R, Martinez E, Sarmento A, Medina JL. Assessment of body fat composition disturbances by bioimpedance analysis in HIV-infected adults. J Endocrinol Investig. 2011;34(10):e321-9.

9. WHO: Physical Status: The Use And Interpretation Of Anthropometry. In Report of a WHO Expert Committee. Geneva: World Health Organization; 1995.

10. Freitas $P$, Carvalho D, Souto S, Santos AC, Xerinda S, Marques R, Martinez E, Sarmento A, Medina JL. Impact of lipodystrophy on the prevalence and components of metabolic syndrome in HIV-infected patients. BMC Infect Dis. 2011:11(1):246.

11. Bonnet E, Delpierre C, Sommet A, Marion-Latard F, Herve R, Aquilina C, Labau E, Obadia M, Marchou B, Massip P, Perret B, Bernard J. Total body composition by DXA of 241 HIV-negative men and 162 HIV-infected men: proposal of reference values for defining lipodystrophy. J Clin Densitom. 2005;8(3):287-92.

12. Freitas $P$, Santos AC, Carvalho D, Pereira J, Marques R, Martinez E, Sarmento A, Medina JL. Fat mass ratio: an objective tool to define lipodystrophy in HIV-infected patients under antiretroviral therapy. J Clin Densitom. 2010; 13(2):197-203.

13. Yoshizumi T, Nakamura T, Yamane M, Islam AH, Menju M, Yamasaki K, Arai T, Kotani K, Funahashi T, Yamashita S, Yoshizumi T, Matsuzawa Y. Abdominal fat: standardised technique for measurement at CT. Radiology. 1999;211(1): 283-6.

14. van der Kooy K, Seidell JC. Techniques for measurement of visceral fat: a practical guide. Int J Obes Relat Metab Disord. 1993:17(4):187-96.

15. World Health Organization: Definition, Diagnosis and Classification Of Diabetes Mellitus And Its Complications. In Report of WHO Consulation. Geneva;1999.

16. Matthews DR, Hosker JP, Rudenski AS, Naylor BA, Treacher DF, Turner RC. Homeostasis model assessment: insulin resistance and beta-cell functions from fasting plasma glucose and insulin concentrations in man. Diabetologia. 1985;28(7):412-9.

17. Third Report of the National Cholesterol Education Program (NCEP) expert panel on detection, evaluation, and treatment of high blood cholesterol in adults (adult treatment panel III) final report. Circulation 2002, P106-P3143.

18. Polak JF, O'Leary DH, Kronmal RA, Wolfson SK, Bond MG, Tracy RP, Gardin JM, Kittner SJ, Price TR, Savage PJ. Sonographic evaluation of carotid artery atherosclerosis in the elderly: relationship of disease severity to stroke and transient ischemic attack. Radiology. 1993;188(2):363-70.

19. O'Leary DH, Polak JF, Kronmal RA, Manolio TA, Burke GL, Wolfson SK Jr. Carotid-artery intima and media thickness as a risk factor for myocardial infarction and stroke in older adults. Cardiovascular health study collaborative research group. N Engl J Med. 1999;340(1):14-22.

20. Mangili A, Jacobson DL, Gerrior J, Polak JF, Gorbach SL, Wanke CA. Metabolic syndrome and subclinical atherosclerosis in patients infected with HIV. Clin Infect Dis. 2007;44(10):1368-74.

21. Grundy SM, Pasternak R, Greenland P, Smith S Jr, Fuster V. Assessment of cardiovascular risk by use of multiple-risk-factor assessment equations: a statement for healthcare professionals from the American Heart Association and the American College of Cardiology. Circulation. 1999 Sep 28;100(13): 1481-92.

22. Rose KA, Vera J, Drivas P, Banya W, Keenan N, Pennell DJ, Winston A. Atherosclerosis is evident in treated HIV-infected subjects with low cardiovascular risk by carotid cardiovascular magnetic resonance. J Acquir Immune Defic Syndr. 2016;71(5):514-21

23. Samanta S, Balasubramanian S, Rajasingh S, Patel U, Dhanasekaran A, Dawn B, Rajasingh J. MicroRNA: a new therapeutic strategy for cardiovascular diseases. Trends Cardiovasc Med. 2016;26(5):407-19.

24. Friis-Moller N, Sabin CA, Weber R, d'Arminio Monforte A, El-Sadr WM, Reiss $P$, Thiebaut $R$, et al. Combination antiretroviral therapy and the risk of myocardial infarction. N Engl J Med. 2003;349(21):1993-2003.

25. Grinspoon S, Carr A, et al. Cardiovascular risk and body-fat abnormalities in HIV-infected adults. N Engl J Med. 2005;352(1):48-62.

26. Chambless LE, Heiss G, Folsom AR, et al. Association of coronary heart disease incidence with carotid arterial wall thickness and major risk factors: the atherosclerosis risk in communities (ARIC) study, 1987-1993. Am J Epidemiol. 1997;146:483-94.

27. Bots ML, Hoes AW, Koudstaal PJ, Hofman A, Grobbee DE. Common carotid intima-media thickness and risk of stroke and myocardial infarction: the Rotterdam study. Circulation. 1997;96:1432-7.

28. Salonen JT, Salonen R. Ultrasound B-mode imaging in observational studies of atherosclerotic progression. Circulation. 1993;87(Suppl 3):II56-65.

29. Stein JH, Currier JS, Hsue PY. Arterial disease in patients with human immunodeficiency virus infection: what has imaging taught us? JACC Cardiovascular imaging. 2014;7(5):515-25.

30. Kaplan RC, Kingsley LA, Gange SJ, Benning L, Jacobson LP, Lazar J, et al. Low CD4+ T-cell count as a major atherosclerosis risk factor in HIV-infected women and men. AIDS. 2008;22(13):1615-24.

31. Mercier $P$, Thiebaut $R$, Lavingnolle $V$, Pellegrin $J$, Yvorra-Vives MC, Morlat $P$, Ragnaud JM, Dupon M, Malvy D, Bellet $H$, et al. Evaluation of cardiovascular risk factors in HIV-1 infected patients using carotid intima-media thickness measurement. Ann Med. 2002;34(1):55-63.

32. Volpe GE, Tang AM, Polak JF, Mangili A, Skinner SC, Wanke CA. Progression of carotid intima media thickness and coronary artery calcium over 6 years in an HIV- infected cohort. J Acquir Immune Defic Syndr. 2013;64:51-7.

33. Hanna DB, Post WS, Deal JA, Hodis HN, Jacobson LP, Mack WJ, et al. HIV infection is associated with progression of subclinical carotid atherosclerosis. Clinical infectious diseases: an official publication of the Infectious Diseases Society of America. 2015;61(4):640-50.

34. Baker JV, Henry WK, Patel $P$, et al. Progression of carotid intima-media thickness in a contemporary human immunodeficiency virus cohort. Clin Infect Dis. 2011:53:826-35.

35. Hsue PY, Hunt PW, Schnell A, et al. Role of viral replication antiretroviral therapy, and immunodeficiency in HIV-associated atherosclerosis. AIDS. 2009;23:1059-67.

36. Sankatsing RR, Wit FW, Vogel M, de Groot E, Brinkman K, Rockstroh JK, Kastelein JJ, Stroes ES, Reiss P. Increased carotid intima-media thickness in HIV patients treated with protease inhibitors as compared to nonnucleoside reverse transcriptase inhibitors. Atherosclerosis. 2009;202(2): 589-95.

37. de Saint ML, Vandhuick O, Guillo P, Bellein V, Bressollette L, Roudaut N, Amaral A, Pasquier E. Premature atherosclerosis in HIV positive patients and cumulate time of exposure to antiretroviral therapy (SHIVA study). Atherosclerosis. 2006;185(2):361-7.

38. Bongiovanni M, Casana M, Cicconi P, Pisacreta M, Codemo R, Pelucchi M, d'Arminio Monforte A, Bini T. Predicteve factors of vascular intima media thickness in HIV positive subjects. J Antimicrob Chemother. 2008; 61(1):195-9. 
39. Py H, Lo JC, Franklin A, Bolger AF, Martin JN, Deeks SG, Walters DD. Progression of atherosclerosis as assessed by carotid intima-media thickness in patients with HIV infection. Circulation. 2004;109(13):1603-8.

40. Huber HB, Feinleib M, McNamara PM, Castelli WP. Obesity as an independent risk factor for cardiovascular disease: a 26-year follow-up of participants in the Framingham heart study. Circulation. 1983;67(5):968-77.

41. Burke GL, Bertoni AG, Shea S, Tracy R, Watson KE, Blumenthal RS, Chung H, Carnethon MR. The impact of obesity on cardiovascular disease risk factors and subclinical vascular disease: the multi-ethnic study of atherosclerosis. Arch Intern Med. 2008;168(9):928-35.

Submit your next manuscript to BioMed Central and we will help you at every step:

- We accept pre-submission inquiries

- Our selector tool helps you to find the most relevant journal

- We provide round the clock customer support

- Convenient online submission

- Thorough peer review

- Inclusion in PubMed and all major indexing services

- Maximum visibility for your research

Submit your manuscript at www.biomedcentral.com/submit
Biomed Central 